\title{
Salt Tolerant Eggplant Rootstocks Modulate Sodium Partitioning in Tomato Scion and Improve Performance under Saline Conditions
}

\author{
Satish Kumar Sanwal ${ }^{1, *}$, Anita Mann ${ }^{1}{ }^{(\mathbb{D}}$, Arvind Kumar ${ }^{1}{ }^{(\mathbb{D}}$, Hari Kesh ${ }^{1}$, Gurpreet Kaur ${ }^{1}$, Arvind Kumar Rai ${ }^{1}$, \\ Raj Kumar ${ }^{1}$, Parbodh C. Sharma ${ }^{1}$, Ashwani Kumar ${ }^{1}{ }^{(D}$, Anant Bahadur ${ }^{2}$, Bijendra Singh ${ }^{2}$ and Pradeep Kumar ${ }^{3, *(D)}$ \\ 1 ICAR-Central Soil Salinity Research Institute, Karnal 132001, Haryana, India; anita.mann@icar.gov.in (A.M.); \\ arvind.kumar2@icar.gov.in (A.K.); harikeshkaul55@gmail.com (H.K.); gurpreetkaurcssri@gmail.com (G.K.); \\ ak.rai@icar.gov.in (A.K.R.); rajkumar2@icar.gov.in (R.K.); Parbodh.Chander@icar.gov.in (P.C.S.); \\ Ashwani.Kumar1@icar.gov.in (A.K.) \\ 2 ICAR-Indian Institute of Vegetable Research, Varanasi 221305, Uttar Pradesh, India; \\ anant.bahadur@icar.gov.in (A.B.); bsinghiivr@gmail.com (B.S.) \\ 3 ICAR-Central Arid Zone Research Institute, Jodhpur 342003, Rajasthan, India \\ * Correspondence: satish.sanwal@icar.gov.in (S.K.S.); pradeep.kumar4@icar.gov.in (P.K.)
}

Citation: Sanwal, S.K.; Mann, A.; Kumar, A.; Kesh, H.; Kaur, G.; Rai, A.K.; Kumar, R.; Sharma, P.C.; Kumar, A.; Bahadur, A.; et al. Salt Tolerant Eggplant Rootstocks Modulate Sodium Partitioning in Tomato Scion and Improve Performance under Saline Conditions. Agriculture 2022, 12, 183. https://doi.org/10.3390/ agriculture12020183

Academic Editor: Yuan Huang

Received: 19 December 2021

Accepted: 24 January 2022

Published: 27 January 2022

Publisher's Note: MDPI stays neutral with regard to jurisdictional claims in published maps and institutional affiliations.

Copyright: (C) 2022 by the authors. Licensee MDPI, Basel, Switzerland. This article is an open access article distributed under the terms and conditions of the Creative Commons Attribution (CC BY) license (https:// creativecommons.org/licenses/by/ $4.0 /)$.

\begin{abstract}
Grafting on salt tolerant eggplant rootstocks can be a promising approach for enhancing the salinity tolerance of tomato. In this study, the performance of tomato cv. Kashi Aman grafted on two salt tolerant eggplant rootstocks (IC-111056 and IC-354557) was evaluated against non-grafted control under saline $\left(\mathrm{EC}_{\mathrm{iw}} 6\right.$ and $\left.9 \mathrm{dS} \mathrm{m}{ }^{-1}\right)$ and non-saline $\left(\mathrm{EC}_{\mathrm{iw}} \sim 1 \mathrm{dS} \mathrm{m} \mathrm{m}^{-1}\right)$ irrigation for 2 years. Grafting improved tomato plant performance under salt stress. Moreover, rootstock IC-111056 outperformed IC-354557. An increase in the average fruit yield of grafted plants compared with non-grafted control at 6 and $9 \mathrm{dS} \mathrm{m}^{-1}$ was $24.41 \%$ and 55.84\%, respectively with rootstock IC-111056 and 20.25\% and $49.08 \%$, respectively with IC-354557. Grafted plants maintained a superior water status under saline irrigation, evidenced with the relative water content and chlorophyll SPAD index, along with higher proline and antioxidant enzyme activities (superoxide dismutase, catalase, and ascorbate peroxidase). Rootstocks mediated the partitioning of toxic saline ions in the scions by promoting higher $\mathrm{Na}^{+}$ accumulation (14\% of mean accumulation) in the older leaves and lower $(24 \%)$ in the younger leaves of grafted plants. This resulted in higher $\mathrm{K}^{+} / \mathrm{Na}^{+}$ratios within the younger (active) leaves of the grafted plants. Our study demonstrates that grafting tomato seedlings on selected salt tolerant eggplant rootstocks is a viable alternative for improving plant physiological status and fruit yield under salt stress, through favorable modulation of salt ion partitioning in the scions.
\end{abstract}

Keywords: tomato grafting; $\mathrm{Na}^{+}$partitioning; salinity tolerance; antioxidant enzymes; fruit yield

\section{Introduction}

Among abiotic stresses, salinity is one of the critical stresses inhibiting plant growth and crop yields. Globally, salt-affected soils represent $7 \%$ of the total area, where saline and alkaline soils constitute about more than 1100 million hectares of land [1]. Salinity has affected approximately 20-33\% of agricultural land across the world [2].

Soil salinity often occurs concomitantly with saline ground water in arid and semi-arid regions, exacerbating the effect on crop growth. Higher levels of salt in soil reduce the productivity of most of the agricultural crops, including vegetables, with the latter as more salt sensitive. The salinity-induced stress can be triggered by the excessive use of poor-quality ground water for irrigation, along with climate change and excessive irrigation associated with intensive farming [3]. The salinity threshold $\left(E_{t}\right)$ of most of the vegetable crops is very low, generally between $\mathrm{EC}_{\mathrm{t}} 1$ to $2.5 \mathrm{dS} \mathrm{m}^{-1}$ [4]. 
Tomato (Solanum lycopersicum L.) is cultivated and consumed as fresh and processed food, and ranks second after potato. Tomatoes are reported as moderately sensitive (threshold limit up to $2.5 \mathrm{dS} \mathrm{m}^{-1}$ ) to salt stress, and thus high salinity can substantially limit the productivity $[5,6]$ through decreased plant height, shoot-root biomass [7], oxidative stress, and inhibition of photosynthesis [8]. The high salinity affects several physiological and biochemical processes due to ion toxicity, which is caused by the high accumulation of $\mathrm{Na}^{+}$ and $\mathrm{Cl}^{-}$ions combined with low levels of $\mathrm{K}^{+}, \mathrm{Ca}^{2+}, \mathrm{SO}_{4}{ }^{2-}$, and $\mathrm{NO}^{3-}$ ions, in addition to osmotic stress $[9,10]$.

Improvement in tomato salt tolerance through modern breeding and biotechnological approaches has been limited since salt tolerance is a complex trait involving several quantitative and environmental factors [11,12]. Although advanced genetic mapping strategies and QTL analysis improved the understanding of the genetics of salt tolerance and related traits, limited success was achieved through marker-assisted selection. The dynamic nature of salinity with respect to time and space, as well as limited experimental designs restrict the complete study of genotype-environment interactions [13]. Therefore, the crop breeding program can be complemented with a suitable management option, such as grafting tomato on appropriate salt tolerant rootstocks [14].

Grafting has been reported as a rapid method for enhancing salt tolerance in vegetable crops [10]. Although grafting was initially used for improving crop tolerance against biotic stress, additional evidence proved the association of grafting with yield improvement under abiotic stresses (salinity, temperature, flooding), and better water and nutrient use efficiency $[15,16]$. Grafting counteracts the salinity effects by maintaining low $\mathrm{Na}^{+} / \mathrm{K}^{+}$ ratios in the shoot and improves leaf stomatal conductance [17]. The behavior of the rootstock in different plant species influences the metabolic processes of the scion leading to tolerance [18].

Most of the Solanaceous crops have been used as a rootstock for tomato cultivation to manage abiotic stress [19]. Solanum habrochaites and other wild species provide a broad spectrum of tolerance [20]. Previously, a tomato scion was grafted on a tomato rootstock for salt stress tolerance [21-23]. However, only a small amount of information is available for salt tolerance of tomato grafted on an eggplant rootstock. Therefore, this study was planned to explore (i) the agronomic performance of high yielding tomato cultivars grafted on two eggplant rootstocks, and (ii) assess the biochemical and physiological changes resulting from scion-rootstock interactions under saline water irrigation.

\section{Materials and Methods}

\subsection{Plant Material and Growth Conditions}

The seeds of eggplant rootstocks IC-354557 and IC-111056 (indigenously collected and registered at the National Bureau of Plant Genetic Resources, New Delhi, India) were sown during the winter season (October) of 2017 and 2018. One week later, tomato seeds of cv. Kashi Aman used as a scion, were sown. These two eggplant rootstocks were reported as tolerant to abiotic stress, specifically waterlogging stress [24] and salinity stress $\left(\mathrm{EC}_{\mathrm{iw}}\right)$ of $9 \mathrm{dS} \mathrm{m}^{-1}$ (our unpublished data). Kashi Aman is a high yielding round-fruited tomato cultivar that is salt sensitive. Single seeds of rootstocks were sown in small disposable $100 \mathrm{~mL}$ plastic cups, while tomato seeds were sown in standard $20 \mathrm{~cm}$ pots. The potting mixture for both species comprised of soil, coco peat, vermiculite, and perlite in 3:1:1:1 ratio. Light irrigations were provided daily and the seedlings of rootstocks and scions were raised for 30 and 23 days, respectively. At this stage, the plants attained the stem thickness of 1.5-3.0 $\mathrm{mm}$ and each plant had at least 2-3 true leaves.

The splice grafting technique was used to graft 23-day-old tomato scions on 30-day-old eggplant rootstocks. About $7 \mathrm{~mm}$ of slanting cut was made in the rootstock and scion to allow for a perfect union. Grafting union was supported with grafting clips and grafted plants were immediately transferred to a grafting chamber with very low light, high humidity (more than $85 \%$ ), and moderate temperature $\left(24-30^{\circ} \mathrm{C}\right)$. After $5-7$ days, the grafted plants were shifted to a polyhouse covered with a shading net for acclimatization. Sprouts 
from rootstocks were removed at regular intervals. Grafted plants were transplanted to pots 17-18 days after grafting.

The grafted and non-grafted tomato plants were transplanted in $24 \mathrm{~cm}$ diameter pots filled with $16 \mathrm{~kg}$ topsoil (sandy loam with $0.45 \%$ organic carbon) during the winter season (20 December) of 2017-2018 and (22 December) of 2018-2019. An estimated dose of fertilizer, i.e., 3.75, 2.0, $2.5 \mathrm{~g}$ of $\mathrm{N}, \mathrm{P}$, and $\mathrm{K}$ was applied. However, half of the quantity of $\mathrm{N}$ and a full dose of $\mathrm{P}$ and $\mathrm{K}$ were added at the time of pot filling. In addition, the remaining quantities of $\mathrm{N}$ were applied in an equal dose at 30 and 60 days after transplanting. Each replicate consisted of nine plants, i.e., three non-grafted tomato plants, and three each of grafted on eggplant rootstocks IC-111056 and IC-354557. Natural saline ground water $\left(\mathrm{EC}_{\mathrm{iw}} \sim 18 \mathrm{dS} \mathrm{m}^{-1}\right)$ available at the Nain experimental farm of the Institute situated at Panipat (Haryana), India was used to prepare the saline water of desired salinity $\left(\mathrm{EC}_{\mathrm{iw}}\right.$ 6 and $9 \mathrm{dS} \mathrm{m}^{-1}$ ) by diluting with good quality water, while for control treatment, the best available water of $\mathrm{EC}_{\mathrm{iw}} \sim 1 \mathrm{dS} \mathrm{m}^{-1}$ was used. Saline ground water of Nain farm had neutral $\mathrm{pH}$ with a dominance of $\mathrm{Na}^{+}, \mathrm{Ca}^{2+}, \mathrm{Mg}^{2+}, \mathrm{Cl}^{-}$, and $\mathrm{SO}_{4}{ }^{2-}$ ions. Saline irrigation for different treatments was first applied 10 days after transplanting. Further irrigation was scheduled based on 100\% evapotranspiration (ET) and 21 irrigations were provided during the whole crop period. At the time of final harvesting, soil samples were collected to measure the build-up of soil salinity in each treatment (Table 1).

Table 1. Soil status: Before and after the experiment.

\begin{tabular}{ccccc}
\hline Parameters & Initial Soil Status & Control & $\mathbf{E C}_{\mathbf{i w}} \mathbf{6} \mathbf{d S} \mathbf{~ m}^{-\mathbf{1}}$ & $\mathbf{E C}_{\mathbf{i w}} \mathbf{9} \mathbf{d S} \mathbf{~ m}^{\mathbf{- 1}}$ \\
\hline & & & \\
\hline $\mathrm{EC}_{\mathrm{e}}$ & 0.35 & 0.42 & 6.14 & 8.86 \\
$\mathrm{pH}_{\mathrm{s}}$ & 7.05 & 7.18 & 7.25 & 7.29 \\
\hline & & & \\
\hline $\mathrm{EC}_{\mathrm{e}}$ & 0.30 & 0.44 & 6.21 & 8.94 \\
$\mathrm{pH}_{\mathrm{s}}$ & 7.08 & 7.24 & 7.31 & 7.38 \\
\hline
\end{tabular}

\subsection{Fruit Yield and Quality Parameters}

The plant height of the three plants from each replicate was measured before the last picking date. When the fruits turned slightly pink or red, they were harvested manually every 3-5 days and the total yield/plant (g) was calculated. The average fruit weight $(\mathrm{g})$ was calculated using the data of 10 fruits from each replicate. Total soluble solids (TSS) of a representative sample size (four fruits per treatment) were measured on a portable hand refractometer (Erma Inc., Tokyo, Japan) as ${ }^{\circ}$ Brix at $20^{\circ} \mathrm{C}$.

\subsection{Physiological and Biochemical Traits}

All of the physiological and biochemical parameters were determined at the onset of flowering. The leaf greenness SPAD index was measured between 09:00 to 11:00 $\mathrm{h}$ using SPAD-502 (Konica Minolta Corp., Solna, Sweden) on the intact top of three fully opened leaves. The relative water content (RWC) was measured in detached third and fourth leaves from the top at 10:00-12:00 $\mathrm{h} \mathrm{[25].}$

$$
\mathrm{RWC}=(\mathrm{FW}-\mathrm{DW}) /(\mathrm{FW}-\mathrm{TW}) \times 100
$$

where FW is the leaf fresh weight, DW is the leaf dry weight, and TW is the turgid leaf weight.

The proline content of fresh leaves was estimated using the ninhydrin reagent [26] and quantified as $\mathrm{mg} \mathrm{g}^{-1}$ fresh weight. Antioxidant enzymes, superoxide dismutase (SOD), and ascorbate peroxidase (APX) were extracted from leaves in a $0.1 \mathrm{M}$ phosphate buffer ( $\mathrm{pH} 7.5)$ consisting of $5 \%(w / v)$ polyvinylpolypyrrolidone, $1 \mathrm{mM}$ EDTA, and $10 \mathrm{mM} \beta-$ mercapto-ethanol, according to the modified method [27]. Peroxidase (POX) was extracted 
in a $0.01 \mathrm{M}$ phosphate buffer ( $\mathrm{pH} 7.5)$ with $3 \%(w / v)$ polyvinylpolypyrrolidone. The SOD enzyme activity was estimated as its ability to inhibit light-induced conversion of nitroblue tetrazolium (NBT) to formazan [28]. APX was quantified as one unit of APX corresponding to 1.0 O.D. change per min [29]. The POX activity was calculated as $1.0 \mu \mathrm{mol}$ of $\mathrm{H}_{2} \mathrm{O}_{2}$ utilized per $\min$ [30]. The catalase (CAT) activity was measured for $1 \mathrm{~min}$ based on the decomposition of $\mathrm{H}_{2} \mathrm{O}_{2}$ at $240 \mathrm{~nm}$ [31].

\subsection{Ionic Content}

$\mathrm{Na}^{+}$and $\mathrm{K}^{+}$contents of leaves and roots were determined at the harvest stage. Properly oven dried and ground fine samples were digested in di-acid mixture for estimation of $\mathrm{Na}^{+}$ and $\mathrm{K}^{+}$contents using the flame photometer (PFP7, Jenway, Bibby Scientific, Stone, UK).

\subsection{Statistical Analysis}

The experiment was conducted in a randomized complete block design replicated five times with three plants per replicate. Morphological and biochemical observations were tested for normality and variance homogeneity through the Shapiro-Wilk test and Levene's test. Additionally, if necessary, appropriate transformations were applied. All of the means of morphological and biochemical traits were compared using the two-way ANOVA (grafted plants $\times$ salinity levels) and repeated measures analysis, by the Type III sum of squares of GLM procedure on SAS (version 9.3, SAS Institute Inc., Cary, NC, USA). ANOVA tables for different parameters are provided in Supplementary Tables S1-S3. To discriminate significant differences between the grafted and non-grafted combinations, the least significant difference (LSD) test was used at probability levels of 0.05 and 0.01 . The $\mathrm{R}$ program was used for the correlation matrix [32] and data were analyzed using Corrplot [33] package.

\section{Results}

\subsection{Yield and Quality Traits}

The fruit yield per plant, average fruit weight, and plant height decreased significantly with gradient salinity levels in both non-grafted and grafted plants, with a pronounced

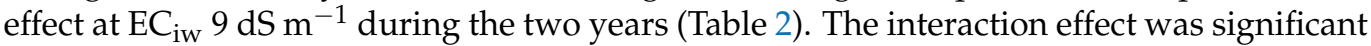
for fruit yield per plant and average fruit weight between the saline treatment and different rootstocks, indicating the differential response of each rootstock to increasing salinity levels. For plant height, the salinity-rootstock interaction was non-significant. During 2017, under control conditions, the yield was at par in plants grafted on rootstock IC-111056 and non-grafted plants, whereas it was significantly reduced $(7.35 \%)$ in plants grafted on rootstock IC-354557. On the contrary, at an increased salinity level of $6 \mathrm{dS} \mathrm{m} \mathrm{m}^{-1}$, plants grafted on rootstocks IC-111056 and IC-354557 produced $24.07 \%$ and $21.08 \%$ higher yield than non-grafted pants. Furthermore, the difference in the fruit yield per plant for both rootstocks was non-significant. As the salinity level further increased to $9 \mathrm{dS} \mathrm{m}{ }^{-1}$, the yield considerably reduced in comparison with control and $6 \mathrm{dS} \mathrm{m} \mathrm{m}^{-1}$ salinity level. However, the fruit yield produced by plants grafted on rootstocks IC-111056 (58.68\%) and IC-354557 $(50.37 \%)$ was significantly more in comparison with non-grafted plants. The fruit yield was $16.74 \%$ more with rootstock IC-111056 than IC-354557.

During 2018, at salinity levels of 6 and $9 \mathrm{dS} \mathrm{m} \mathrm{m}^{-1}$, grafted plants on rootstock IC-111056 produced higher yield of $24.75 \%$ and $53 \%$, respectively, whereas on rootstock IC-354557 the grafted plants produced higher yield of $19.43 \%$ and $47.78 \%$, respectively than non-grafted plants. The yield was higher by $6.6 \%$ and $10 \%$, respectively with rootstock IC-111056 than IC-354557 at the salinity level of 6 and $9 \mathrm{dS} \mathrm{m}^{-1}$ (Table 2). Similarly, the plants grafted on rootstock IC-111056 performed better for the average fruit weight at different salinity levels. However, total soluble solids were observed as significantly higher in the year 2017 only at different salinity treatments. 
Table 2. Effect of salinity and rootstock combinations on yield components and physiological parameters of tomato (cv. Kashi Aman).

\begin{tabular}{|c|c|c|c|c|c|c|c|}
\hline $\begin{array}{l}\text { Salinity } \\
\text { Treatment } \\
\left(\mathrm{dS} \mathrm{m}^{-1}\right)\end{array}$ & Rootstock & $\begin{array}{l}\text { Plant Height } \\
\text { (cm) }\end{array}$ & $\begin{array}{l}\text { Fruit Yield } \\
\left(\text { g plant }^{-1}\right)\end{array}$ & $\begin{array}{l}\text { Av. Fruit wt. } \\
\text { (g) }\end{array}$ & $\begin{array}{c}\text { TSS } \\
\left({ }^{\circ} \text { Brix }\right)\end{array}$ & $\begin{array}{l}\text { SPAD } \\
\text { Index }\end{array}$ & $\begin{array}{c}\text { RWC } \\
(\%)\end{array}$ \\
\hline \multicolumn{8}{|c|}{2017} \\
\hline \multirow[t]{3}{*}{ Control } & Non-grafted & $64.33 a$ & $2577.3 a$ & $64.25 a$ & $4.35 \mathrm{a}$ & $60.62 b$ & $83.30 \mathrm{a}$ \\
\hline & IC-111056 & $69.23 a$ & $2518.0 \mathrm{a}$ & $63.12 \mathrm{a}$ & $4.40 \mathrm{a}$ & $62.63 a$ & $82.31 \mathrm{a}$ \\
\hline & IC-354557 & $62.90 \mathrm{a}$ & $2388.0 \mathrm{~b}$ & $63.83 a$ & $4.38 \mathrm{a}$ & $62.39 \mathrm{ab}$ & $83.06 \mathrm{a}$ \\
\hline \multirow[t]{3}{*}{$\mathrm{EC}_{\mathrm{iw}} 6$} & Non-grafted & $58.33 a$ & $1420.8 b$ & $56.10 \mathrm{~b}$ & $5.10 \mathrm{a}$ & $58.79 c$ & $70.91 \mathrm{a}$ \\
\hline & IC-111056 & $60.66 a$ & $1871.3 a$ & $59.23 a$ & $5.14 \mathrm{a}$ & $60.47 a$ & $76.63 a$ \\
\hline & IC-354557 & $57.66 \mathrm{a}$ & $1800.4 \mathrm{a}$ & $60.22 \mathrm{a}$ & $5.16 \mathrm{a}$ & $59.51 b$ & $76.12 \mathrm{a}$ \\
\hline \multirow[t]{3}{*}{$\mathrm{EC}_{\mathrm{iw}} 9$} & Non-grafted & $48.55 a$ & $522.8 \mathrm{c}$ & $41.37 \mathrm{~b}$ & $4.45 \mathrm{a}$ & $50.77 \mathrm{c}$ & $64.82 \mathrm{a}$ \\
\hline & IC-111056 & $55.42 \mathrm{a}$ & $1265.3 a$ & $48.63 a$ & $4.32 b$ & $57.19 a$ & $71.20 \mathrm{a}$ \\
\hline & IC-354557 & $51.88 \mathrm{a}$ & $1053.4 b$ & $47.62 \mathrm{a}$ & $4.36 \mathrm{~b}$ & $54.07 \mathrm{~b}$ & $70.03 \mathrm{a}$ \\
\hline \multicolumn{8}{|c|}{ Significance } \\
\hline \multicolumn{2}{|c|}{ Salinity } & $* *$ & $* *$ & $* *$ & $* *$ & $* *$ & $* *$ \\
\hline \multicolumn{2}{|c|}{ Rootstock } & $* *$ & $* *$ & $* *$ & ns & $* *$ & ns \\
\hline \multicolumn{2}{|c|}{ Salinity $\times$ rootstock } & ns & $* *$ & $* *$ & $* *$ & $* *$ & ns \\
\hline \multicolumn{8}{|c|}{2018} \\
\hline \multirow[t]{3}{*}{ Control } & Non-grafted & $64.88 \mathrm{a}$ & $2243.10 \mathrm{~b}$ & $60.15 b$ & $4.46 a$ & $58.21 \mathrm{~b}$ & $83.44 a$ \\
\hline & IC-111056 & $67.35 \mathrm{a}$ & $2301.87 \mathrm{a}$ & $61.87 \mathrm{a}$ & $4.42 \mathrm{a}$ & $59.35 a$ & $82.63 \mathrm{a}$ \\
\hline & IC-354557 & $63.97 a$ & $2227.15 b$ & $62.05 \mathrm{a}$ & $4.35 \mathrm{a}$ & $58.54 a$ & $84.68 \mathrm{a}$ \\
\hline \multirow[t]{3}{*}{$\mathrm{EC}_{\mathrm{iw}} 6$} & Non-grafted & $56.48 \mathrm{a}$ & $1340.77 \mathrm{c}$ & $54.30 \mathrm{a}$ & $5.16 a$ & $55.09 \mathrm{~b}$ & $72.54 \mathrm{a}$ \\
\hline & IC-111056 & $58.62 \mathrm{a}$ & $1781.70 \mathrm{a}$ & $55.82 \mathrm{a}$ & $5.08 \mathrm{a}$ & $57.47 \mathrm{a}$ & $77.10 \mathrm{a}$ \\
\hline & IC-354557 & $56.33 a$ & $1664.05 b$ & $54.53 a$ & $5.14 \mathrm{a}$ & $56.17 \mathrm{~b}$ & $77.78 \mathrm{a}$ \\
\hline \multirow[t]{3}{*}{$\mathrm{EC}_{\mathrm{iw}} 9$} & Non-grafted & $48.37 \mathrm{a}$ & $532.18 c$ & $39.00 \mathrm{c}$ & $4.37 \mathrm{a}$ & $49.42 c$ & $66.45 \mathrm{a}$ \\
\hline & IC-111056 & $52.82 \mathrm{a}$ & $1132.25 \mathrm{a}$ & $46.53 a$ & $4.25 \mathrm{a}$ & $54.98 \mathrm{a}$ & $72.86 a$ \\
\hline & IC-354557 & $50.55 a$ & $1019.10 b$ & $43.52 b$ & $4.31 \mathrm{a}$ & $52.67 \mathrm{~b}$ & $71.35 \mathrm{a}$ \\
\hline \multicolumn{8}{|c|}{ Significance } \\
\hline \multicolumn{2}{|c|}{ Salinity } & $* *$ & $* *$ & $* *$ & $* *$ & ** & ** \\
\hline \multicolumn{2}{|c|}{ Rootstock } & $* *$ & $* *$ & $* *$ & ns & $* *$ & ns \\
\hline \multicolumn{2}{|c|}{ Salinity $\times$ rootstock } & ns & $* *$ & $* *$ & ns & $* *$ & ns \\
\hline
\end{tabular}

Means followed by different letters within a column and under a specific treatment effect are significantly different at $p=0.05$ by the least significant difference (LSD) test; $\mathrm{ns},{ }^{* *}$ non-significant or significant at $p=0.01$, respectively.

\subsection{Physiological Traits}

The SPAD index and RWC were significantly affected by different levels of salinity, while a significant interaction effect with rootstock alone was observed for SPAD index only (Table 2). The SPAD index and RWC were highest in plants under control condition followed by plants stressed with saline water at 6 and $9 \mathrm{dS} \mathrm{m}^{-1}$ salt concentrations. Grafted plants on rootstocks IC-111056 and IC-354557 had more leaf greenness SPAD index than non-grafted rootstocks (Kashi Aman) under control, as well as at $\mathrm{EC}_{\mathrm{iw}} 6$ and $9 \mathrm{dS} \mathrm{m}^{-1}$. The SPAD index values of $1.58-2.26 \%$ and $4.20-5.46 \%$, respectively were significantly higher for rootstock IC-111056 than IC-354557 at 6 and $9 \mathrm{dS} \mathrm{m}^{-1}$, during the two years.

\subsection{Biochemical Traits}

The relative concentrations of proline and the activities of CAT, APX, SOD, and POX enzymes were significantly affected by gradient salinity treatments (Table 3 ). The grafted and non-grafted plants showed higher proline content, as well as CAT, APX, SOD, and POX activities under salinity treatments, although they were significantly enhanced in grafted plants only. For proline content, differences between the two rootstocks were significant for $9 \mathrm{dS} \mathrm{m}^{-1}$ treatment only. At $9 \mathrm{dS} \mathrm{m}{ }^{-1}$, higher APX and CAT activities of $11-14 \%$ and $13-14 \%$ were observed in rootstock IC-111056 grafted plants than non-grafted plants in both years. No significant differences were seen in POX and SOD activities between the 
grafted and non-grafted plants in control condition. However, under salt treatment, the grafted plants showed significantly higher SOD and POX activities than non-grafted plants (Table 3).

Table 3. Effect of salinity and rootstock combinations on biochemical parameters of tomato (cv. Kashi Aman).

\begin{tabular}{|c|c|c|c|c|c|c|}
\hline $\begin{array}{c}\text { Salinity } \\
\text { Treatment }\end{array}$ & Rootstock & Proline & CAT & APX & SOD & POX \\
\hline$\left(\mathrm{dS} \mathrm{m}^{-1}\right)$ & & $\left(\mu g^{-1} F W\right)$ & \multicolumn{4}{|c|}{ (Units $\left.\mathrm{g}^{-1} \mathrm{FW}\right)$} \\
\hline \multicolumn{7}{|c|}{2017} \\
\hline \multirow[t]{3}{*}{ Control } & Non-grafted & $387.63 a$ & $12.86 b$ & $75.50 \mathrm{~b}$ & $185.83 a$ & $25.23 a$ \\
\hline & IC-111056 & $364.38 \mathrm{~b}$ & $13.68 \mathrm{ab}$ & $77.64 \mathrm{a}$ & $173.40 \mathrm{a}$ & $26.07 a$ \\
\hline & IC-354557 & $337.51 \mathrm{c}$ & $14.38 \mathrm{a}$ & $75.96 b$ & $181.73 a$ & $23.83 a$ \\
\hline \multirow[t]{3}{*}{$\mathrm{EC}_{\mathrm{iw}} 6$} & Non-grafted & $754.17 \mathrm{~b}$ & $13.32 \mathrm{c}$ & $145.80 \mathrm{~b}$ & $246.87 b$ & $32.07 \mathrm{~b}$ \\
\hline & IC-111056 & $943.73 a$ & $16.13 a$ & $160.20 \mathrm{a}$ & $271.03 a$ & $39.67 a$ \\
\hline & IC-354557 & $936.53 a$ & $15.42 b$ & $156.40 \mathrm{a}$ & $236.13 a$ & $38.70 \mathrm{a}$ \\
\hline \multirow[t]{3}{*}{$\mathrm{EC}_{\mathrm{iw}} 9$} & Non-grafted & $991.25 c$ & $15.36 \mathrm{c}$ & $157.24 \mathrm{c}$ & $294.13 c$ & $46.13 b$ \\
\hline & IC-111056 & $1324.80 \mathrm{a}$ & $17.84 \mathrm{a}$ & $176.30 \mathrm{a}$ & $333.33 a$ & $56.57 \mathrm{a}$ \\
\hline & IC-354557 & $1110.03 b$ & $16.61 b$ & $168.45 b$ & $315.43 b$ & $54.70 \mathrm{a}$ \\
\hline \multicolumn{7}{|c|}{ Significance } \\
\hline \multicolumn{2}{|c|}{ Salinity } & $* *$ & ** & $* *$ & $* *$ & $* *$ \\
\hline \multicolumn{2}{|c|}{ Rootstock } & $* *$ & $* *$ & $* *$ & $* *$ & $* *$ \\
\hline \multicolumn{2}{|c|}{ Salinity $\times$ rootstock } & $* *$ & $* *$ & $* *$ & $* *$ & $* *$ \\
\hline \multicolumn{7}{|c|}{2018} \\
\hline \multirow[t]{3}{*}{ Control } & Non-grafted & $346.40 \mathrm{a}$ & $14.27 \mathrm{~b}$ & $68.30 \mathrm{~b}$ & $196.03 a$ & $22.25 a$ \\
\hline & IC-111056 & $351.39 a$ & $14.07 \mathrm{~b}$ & $70.90 a$ & 190.90a & $21.05 a$ \\
\hline & IC-354557 & $353.90 \mathrm{a}$ & $15.13 a$ & $70.30 \mathrm{a}$ & $188.60 \mathrm{a}$ & $20.47 a$ \\
\hline \multirow[t]{3}{*}{$\mathrm{EC}_{\mathrm{iw}} 6$} & Non-grafted & $709.03 b$ & $15.22 b$ & $136.95 b$ & $245.13 b$ & $31.30 \mathrm{~b}$ \\
\hline & IC-111056 & $920.40 a$ & $17.46 \mathrm{a}$ & $148.32 \mathrm{a}$ & $266.07 a$ & $41.57 \mathrm{a}$ \\
\hline & IC-354557 & $898.45 a$ & $17.25 \mathrm{a}$ & $145.70 a$ & $258.73 a$ & $39.12 b$ \\
\hline \multirow[t]{3}{*}{$\mathrm{EC}_{\mathrm{iw}} 9$} & Non-grafted & $1009.15 c$ & $16.51 \mathrm{c}$ & $144.30 \mathrm{c}$ & $295.57 b$ & $44.27 \mathrm{~b}$ \\
\hline & IC-111056 & $1343.61 \mathrm{a}$ & $19.32 \mathrm{a}$ & $167.50 a$ & $266.30 a$ & $59.48 \mathrm{a}$ \\
\hline & IC-354557 & $1123.75 b$ & $18.81 b$ & $160.30 \mathrm{~b}$ & $314.57 a$ & $55.18 \mathrm{a}$ \\
\hline \multicolumn{7}{|c|}{ Significance } \\
\hline \multicolumn{2}{|c|}{ Salinity } & $* *$ & $* *$ & $* *$ & $* *$ & $* *$ \\
\hline \multicolumn{2}{|c|}{ Rootstock } & $* *$ & $* *$ & $* *$ & $* *$ & $* *$ \\
\hline \multicolumn{2}{|c|}{ Salinity $\times$ rootstock } & $* *$ & $* *$ & $* *$ & * & $*$ \\
\hline
\end{tabular}

Means followed by different letters within a column and under a specific treatment effect are significantly different at $p=0.05$ by the least significant difference (LSD) test; ${ }^{*},{ }^{* *}$ significant at $p=0.05$ or 0.01 , respectively.

\subsection{Ionic Content and Ion Partitioning}

Salinity and rootstock combinations significantly affected $\mathrm{Na}^{+}$and $\mathrm{K}^{+}$contents and $\mathrm{Na}^{+} / \mathrm{K}^{+}$ratios in both roots and shoots along with the salinity-rootstock interaction (Table 4). With the increase in salinity, a significant increase in $\mathrm{Na}^{+}$content in the roots and shoots, while a decrease in $\mathrm{K}^{+}$content and $\mathrm{K}^{+} / \mathrm{Na}^{+}$ratio was observed in both grafted and non-grafted plants (Table 4). In 2017, for the $6 \mathrm{dS} \mathrm{m}^{-1}$ salt treatment, the $\mathrm{Na}^{+}$content in shoots was lower by $9.98 \%$ and $14.61 \%$, respectively in plants grafted on rootstocks IC-111056 and IC-354557, whereas the concentration in roots was lower by $5.65 \%$ and $5.05 \%$, respectively. Similarly, during 2018 , the $\mathrm{Na}^{+}$content was lower by $7.91 \%$ and $10.79 \%$, respectively in shoots and lower by $10.62 \%$ and $5.01 \%$, respectively in roots of IC- 111056 and IC-354557 grafted plants than non-grafted plants. In comparison, at the $9 \mathrm{dS} \mathrm{m}^{-1}$ salinity level, the $\mathrm{Na}^{+}$content in shoots of IC-111056 and IC-354557 grafted plants was lower by $3.40 \%$ and $2.61 \%$, respectively than non-grafted plants in 2017 and lower by $2.43 \%$ and $2.19 \%$, respectively in 2018 . Whereas, the $\mathrm{Na}^{+}$content in roots of IC-111056 and IC-354557 
grafted plants was lower by $12.03 \%$ and $5.84 \%$, respectively than non-grafted plants in 2017 and lower by $10.03 \%$ and $3.05 \%$, respectively in 2018 .

Table 4. Effect of salinity treatments on $\mathrm{Na}^{+}$and $\mathrm{K}^{+}$contents as well as $\mathrm{K}^{+} / \mathrm{Na}^{+}$ratios of root and shoot parts of grafted and non-grafted tomato plant (cv. Kashi Aman).

\begin{tabular}{|c|c|c|c|c|c|c|c|}
\hline \multirow{3}{*}{$\begin{array}{c}\begin{array}{c}\text { Salinity } \\
\text { Treatment }\end{array} \\
\left(\mathrm{dS} \mathrm{m} \mathrm{m}^{-1}\right)\end{array}$} & \multirow{3}{*}{ Rootstock } & & & \multicolumn{4}{|c|}{ Element (mg g $\left.{ }^{-1} \mathrm{DW}\right)$} \\
\hline & & \multicolumn{2}{|c|}{$\mathrm{Na}^{+}$} & \multicolumn{2}{|c|}{$\mathbf{K}^{+}$} & \multicolumn{2}{|c|}{$\mathrm{K}^{+} / \mathrm{Na}^{+}$} \\
\hline & & Root & Shoot & Root & Shoot & Root & Shoot \\
\hline \multicolumn{8}{|c|}{2017} \\
\hline \multirow[t]{3}{*}{ Control } & Non-grafted & $4.75 b$ & $4.20 \mathrm{a}$ & $17.02 \mathrm{~b}$ & $13.32 \mathrm{a}$ & $3.58 b$ & $3.17 \mathrm{a}$ \\
\hline & IC-111056 & $4.85 \mathrm{a}$ & $4.17 \mathrm{a}$ & $18.05 a$ & $13.56 a$ & $3.72 \mathrm{a}$ & $3.25 a$ \\
\hline & IC-354557 & $4.65 c$ & $4.16 \mathrm{a}$ & $17.24 b$ & $12.22 b$ & $3.71 \mathrm{a}$ & $2.94 b$ \\
\hline \multirow[t]{3}{*}{$\mathrm{EC}_{\mathrm{iw}} 6$} & Non-grafted & $8.32 \mathrm{a}$ & $5.61 \mathrm{a}$ & $15.31 b$ & $11.77 \mathrm{~b}$ & $1.84 \mathrm{c}$ & $2.10 \mathrm{~b}$ \\
\hline & IC-111056 & $7.85 b$ & $5.05 \mathrm{~b}$ & $15.86 a$ & $12.44 a$ & $2.02 \mathrm{a}$ & $2.46 a$ \\
\hline & IC-354557 & $7.90 \mathrm{~b}$ & $4.79 c$ & $15.23 b$ & $11.89 \mathrm{~b}$ & $1.93 b$ & $2.48 \mathrm{a}$ \\
\hline \multirow[t]{3}{*}{$\mathrm{EC}_{\mathrm{iw}} 9$} & Non-grafted & $11.64 a$ & $8.81 \mathrm{a}$ & $10.72 b$ & $9.89 \mathrm{~b}$ & $0.92 \mathrm{c}$ & $1.12 b$ \\
\hline & IC-111056 & $10.24 \mathrm{c}$ & $8.51 b$ & $11.27 \mathrm{a}$ & $10.20 \mathrm{a}$ & $1.10 \mathrm{a}$ & $1.20 \mathrm{a}$ \\
\hline & IC-354557 & $10.96 b$ & $8.58 b$ & $11.25 a$ & $9.77 \mathrm{~b}$ & $1.03 b$ & $1.14 \mathrm{ab}$ \\
\hline \multicolumn{8}{|c|}{ Significance } \\
\hline \multicolumn{2}{|c|}{ Salinity } & $* *$ & $* *$ & $* *$ & $* *$ & $* *$ & $* *$ \\
\hline \multicolumn{2}{|c|}{ Rootstock } & $* *$ & $* *$ & $* *$ & $* *$ & $* *$ & $* *$ \\
\hline \multicolumn{2}{|c|}{ Salinity $\times$ rootstock } & $* *$ & $* *$ & $* *$ & $* *$ & $* *$ & $* *$ \\
\hline \multicolumn{8}{|c|}{2018} \\
\hline \multirow[t]{3}{*}{ Control } & Non-grafted & $4.82 \mathrm{a}$ & $4.18 \mathrm{~b}$ & $16.66 \mathrm{c}$ & $13.40 \mathrm{a}$ & $3.21 \mathrm{a}$ & $3.46 \mathrm{~b}$ \\
\hline & IC-111056 & $4.93 a$ & $4.26 \mathrm{a}$ & $17.84 \mathrm{a}$ & $13.46 a$ & $3.16 b$ & $3.62 a$ \\
\hline & IC-354557 & $4.86 \mathrm{a}$ & $4.30 \mathrm{a}$ & $17.46 \mathrm{~b}$ & $13.12 b$ & $3.05 c$ & $3.59 a$ \\
\hline \multirow[t]{3}{*}{$\mathrm{EC}_{\mathrm{iw}} 6$} & Non-grafted & $8.19 a$ & $5.56 a$ & $15.12 \mathrm{c}$ & $11.58 \mathrm{c}$ & $2.08 \mathrm{~b}$ & $1.85 \mathrm{c}$ \\
\hline & IC-111056 & $7.32 \mathrm{c}$ & $5.12 b$ & $15.48 \mathrm{a}$ & $12.28 \mathrm{a}$ & $2.40 \mathrm{a}$ & $2.11 a$ \\
\hline & IC-354557 & $7.78 \mathrm{~b}$ & $4.96 \mathrm{~b}$ & $15.32 b$ & $12.05 \mathrm{~b}$ & $2.43 a$ & $1.97 \mathrm{~b}$ \\
\hline \multirow[t]{3}{*}{$\mathrm{EC}_{\mathrm{iw}} 9$} & Non-grafted & 11.16a & $8.64 b$ & $10.56 \mathrm{~b}$ & $9.78 c$ & $1.13 b$ & $0.95 c$ \\
\hline & IC-111056 & $10.04 c$ & $8.43 a$ & $11.24 a$ & $10.08 \mathrm{a}$ & $1.20 \mathrm{a}$ & $1.12 \mathrm{a}$ \\
\hline & IC-354557 & $10.82 b$ & $8.45 a$ & $11.18 \mathrm{a}$ & $9.92 b$ & $1.17 \mathrm{ab}$ & $1.03 \mathrm{~b}$ \\
\hline \multicolumn{8}{|c|}{ Significance } \\
\hline \multicolumn{2}{|c|}{ Salinity } & $* *$ & $* *$ & $* *$ & $* *$ & $* *$ & $* *$ \\
\hline \multicolumn{2}{|c|}{ Rootstock } & $* *$ & $* *$ & $* *$ & $* *$ & $* *$ & * \\
\hline \multicolumn{2}{|c|}{ Salinity $\times$ rootstock } & $* *$ & $* *$ & $* *$ & $* *$ & $* *$ & $* *$ \\
\hline
\end{tabular}

Means followed by different letters within a column and under a specific treatment effect are significantly different at $p=0.05$ by the least significant difference (LSD) test; ${ }^{*}{ }^{* *}$ significant at $p=0.05$ or 0.01 , respectively.

The roots and shoots of tomato plants, grafted on rootstock IC-111056 showed a significantly higher amount of $\mathrm{K}^{+}$content than the plants grafted on IC-354557 and nongrafted plants under salt treatment $\left(\mathrm{EC}_{\mathrm{iw}} 6\right.$ and $9 \mathrm{dS} \mathrm{m}^{-1}$ ) (Table 4). Although, in grafted plants, at salinity of 6 and $9 \mathrm{dS} \mathrm{m}^{-1}$, higher $\mathrm{K}^{+} / \mathrm{Na}^{+}$ratios were found in roots and shoots, except in roots at $\mathrm{EC}_{\mathrm{iw}} 9 \mathrm{dS} \mathrm{m}^{-1}$.

The $\mathrm{Na}^{+}$and $\mathrm{K}^{+}$contents as well as the $\mathrm{K}^{+} / \mathrm{Na}^{+}$ratios in leaves were significantly affected by salinity level, rootstock combinations, leaf orientation, salinity $\times$ rootstock, salinity $\times$ leaf orientation, rootstock $\times$ leaf orientation, year $\times$ leaf orientation, year $\times$ salinity $\times$ leaf orientation during the two seasons. As the salinity level increased, a respective increase in $\mathrm{Na}^{+}$content as well as a decrease in $\mathrm{K}^{+}$content and $\mathrm{K}^{+} / \mathrm{Na}^{+}$ratios in bottom (BL), middle (ML), and upper (UL) leaves were observed during the two seasons (Table 5). 
Table 5. Effect of salinity and rootstock on ion partitioning in leaves orientation.

\begin{tabular}{|c|c|c|c|c|c|c|c|c|c|c|}
\hline \multirow{2}{*}{$\begin{array}{l}\text { Salinity Level } \\
\quad\left(\mathrm{d} S \mathrm{~m}^{-1}\right)\end{array}$} & \multirow{2}{*}{ Rootstock } & \multicolumn{3}{|c|}{$\mathrm{Na}^{+}\left(\mathrm{mg} \mathrm{g}^{-1} \mathrm{DW}\right)$} & \multicolumn{3}{|c|}{$\mathrm{K}^{+}\left(\mathrm{mg} \mathrm{g}^{-1} \mathrm{DW}\right)$} & \multicolumn{3}{|c|}{$\mathrm{K}^{+} / \mathrm{Na}^{+}$} \\
\hline & & BL & ML & UL & BL & ML & UL & BL & ML & UL \\
\hline \multicolumn{11}{|c|}{2017} \\
\hline \multirow[t]{3}{*}{ Control } & Non-grafted & $4.24 \mathrm{aB}$ & $4.06 \mathrm{aB}$ & $3.88 \mathrm{aA}$ & $10.37 \mathrm{bC}$ & $16.56 \mathrm{bB}$ & $20.54 \mathrm{cA}$ & $2.45 \mathrm{aC}$ & $4.08 \mathrm{cB}$ & $5.29 \mathrm{cA}$ \\
\hline & IC-111056 & $4.30 \mathrm{aC}$ & $3.91 \mathrm{aB}$ & $3.35 \mathrm{bA}$ & $10.44 b C$ & $16.19 \mathrm{cB}$ & $29.01 \mathrm{aA}$ & $2.43 a C$ & $4.14 \mathrm{bB}$ & $6.57 \mathrm{aA}$ \\
\hline & IC-354557 & $4.47 \mathrm{aC}$ & $3.98 \mathrm{aAB}$ & $3.69 \mathrm{aA}$ & $10.68 \mathrm{aC}$ & $17.13 \mathrm{aB}$ & $28.29 \mathrm{bA}$ & $2.39 \mathrm{aC}$ & $4.30 \mathrm{aB}$ & $5.77 \mathrm{bA}$ \\
\hline \multirow[t]{3}{*}{$\mathrm{EC}_{\mathrm{iw}} 6$} & Non-grafted & $6.22 \mathrm{cC}$ & $5.38 \mathrm{aB}$ & 4.47aA & $9.74 \mathrm{aC}$ & $14.49 \mathrm{bB}$ & $18.61 \mathrm{cA}$ & $1.57 \mathrm{aC}$ & $2.69 \mathrm{bB}$ & $3.72 \mathrm{cA}$ \\
\hline & IC-111056 & $7.29 \mathrm{aC}$ & $5.17 \mathrm{bB}$ & $3.80 \mathrm{cA}$ & $9.27 \mathrm{bC}$ & $14.91 \mathrm{aB}$ & $22.08 \mathrm{aA}$ & $1.27 \mathrm{cC}$ & $2.88 \mathrm{aB}$ & $4.76 \mathrm{aA}$ \\
\hline & IC-354557 & $6.89 \mathrm{bC}$ & $5.50 \mathrm{aAB}$ & $3.96 \mathrm{bA}$ & $9.75 \mathrm{aC}$ & $15.12 \mathrm{aB}$ & $20.40 \mathrm{bA}$ & $1.42 \mathrm{bC}$ & $2.75 \mathrm{bB}$ & $4.56 \mathrm{bA}$ \\
\hline \multirow[t]{3}{*}{$\mathrm{EC}_{\mathrm{iw}} 9$} & Non-grafted & $8.06 \mathrm{cC}$ & 7.64aB & $6.84 \mathrm{aA}$ & $8.93 \mathrm{aC}$ & $12.01 \mathrm{cB}$ & $13.55 \mathrm{cA}$ & $1.11 \mathrm{aC}$ & $1.31 \mathrm{bB}$ & $1.69 \mathrm{cA}$ \\
\hline & IC-111056 & $9.88 \mathrm{aC}$ & $7.04 \mathrm{bB}$ & $5.06 \mathrm{cA}$ & $8.29 c C$ & $13.19 \mathrm{aB}$ & $17.77 \mathrm{aA}$ & $0.84 \mathrm{abC}$ & $1.59 \mathrm{aB}$ & $2.72 \mathrm{aA}$ \\
\hline & IC-354557 & $9.21 b C$ & $7.72 \mathrm{aB}$ & $5.69 \mathrm{bA}$ & $8.58 \mathrm{bC}$ & $12.39 \mathrm{bB}$ & $15.93 \mathrm{bA}$ & $0.93 \mathrm{aC}$ & $1.60 \mathrm{aB}$ & $2.27 \mathrm{bA}$ \\
\hline \multicolumn{11}{|c|}{2018} \\
\hline \multirow[t]{3}{*}{ Control } & Non-grafted & $4.38 \mathrm{bC}$ & $4.04 \mathrm{bB}$ & $3.76 \mathrm{aA}$ & $11.06 \mathrm{aC}$ & $16.21 \mathrm{aB}$ & $19.42 \mathrm{cA}$ & $2.53 \mathrm{aC}$ & $4.01 \mathrm{aB}$ & $5.16 \mathrm{cA}$ \\
\hline & IC-111056 & $4.62 \mathrm{aC}$ & $4.16 \mathrm{aB}$ & $3.29 \mathrm{cA}$ & $10.94 \mathrm{aC}$ & $15.97 \mathrm{bB}$ & $26.30 \mathrm{aA}$ & $2.37 \mathrm{abC}$ & $3.84 \mathrm{bB}$ & $6.47 \mathrm{aA}$ \\
\hline & IC-354557 & $4.48 \mathrm{bC}$ & $4.03 \mathrm{bB}$ & $3.42 \mathrm{bA}$ & $10.88 \mathrm{aC}$ & $16.27 \mathrm{aB}$ & $25.52 \mathrm{bA}$ & $2.43 \mathrm{aC}$ & $4.04 \mathrm{aB}$ & $6.00 \mathrm{bA}$ \\
\hline \multirow[t]{3}{*}{$\mathrm{EC}_{\mathrm{iw}} 6$} & Non-grafted & $6.30 \mathrm{cC}$ & $5.24 \mathrm{aB}$ & $4.93 \mathrm{aA}$ & $9.95 \mathrm{aC}$ & $14.69 \mathrm{aB}$ & $16.34 \mathrm{cA}$ & $1.58 \mathrm{aC}$ & $2.80 \mathrm{bB}$ & $3.31 \mathrm{cA}$ \\
\hline & IC-111056 & $7.42 \mathrm{aC}$ & $5.32 \mathrm{aB}$ & $3.71 \mathrm{cA}$ & $9.44 c \mathrm{C}$ & $14.89 \mathrm{aB}$ & $23.10 \mathrm{aA}$ & $1.27 \mathrm{bC}$ & $2.80 \mathrm{bB}$ & $4.85 \mathrm{aA}$ \\
\hline & IC-354557 & $7.02 \mathrm{bC}$ & $5.16 \mathrm{aB}$ & $4.02 \mathrm{bA}$ & $9.66 \mathrm{bC}$ & $14.74 \mathrm{aB}$ & $21.33 \mathrm{bA}$ & $1.38 \mathrm{bC}$ & $2.86 \mathrm{aB}$ & $4.31 \mathrm{bA}$ \\
\hline \multirow{3}{*}{$\mathrm{EC}_{\mathrm{iw}} 9$} & Non-grafted & $7.98 \mathrm{cC}$ & 7.54aB & $7.28 \mathrm{aA}$ & $8.74 \mathrm{aB}$ & $12.26 \mathrm{cA}$ & $12.08 \mathrm{cA}$ & $1.10 \mathrm{aC}$ & $1.36 \mathrm{bB}$ & $1.66 \mathrm{cA}$ \\
\hline & IC-111056 & $8.79 \mathrm{aC}$ & $6.92 \mathrm{cB}$ & $5.17 \mathrm{cA}$ & $8.42 b C$ & $13.08 \mathrm{aB}$ & $17.32 \mathrm{aA}$ & $0.96 \mathrm{bC}$ & $1.75 \mathrm{aB}$ & $2.58 \mathrm{aA}$ \\
\hline & IC-354557 & $8.24 b C$ & $7.22 \mathrm{bB}$ & $6.14 \mathrm{bA}$ & $8.54 \mathrm{abC}$ & $12.48 \mathrm{bB}$ & $16.14 \mathrm{bA}$ & $1.04 \mathrm{abC}$ & $1.68 \mathrm{aB}$ & $2.14 \mathrm{bA}$ \\
\hline
\end{tabular}

ANOVA

\section{Salinity}

Rootstock

Leaf orient

Salinity $\times$ Rootstock

Salinity $\times$ Leaf orient

Rootstock $\times$ leaf orient

Year $\times$ Salinity

Year $\times$ Rootstock

Year $\times$ leaf orient

Salinity $\times$ Rootstock $\times$ leaf orient

Year $\times$ Salinity $\times$ Rootstock

Year $\times$ Salinity $\times$ leaf orient

Year $\times$ Rootstock $\times$ leaf orient

Year $\times$ Salinity $\times$ Rootstock $\times$ leaf orient

$\begin{array}{lcc}* * * & * * * & * * * \\ * * * & * * * & * * * \\ * * * & * * * & * * * \\ * & * * * & * * * \\ * * * & * * * & * * * \\ * * * & * * * & * * * \\ \mathrm{~ns} & * * * & * * * \\ * & \mathrm{~ns} & * \\ * & * * * & * * * \\ * * * & * * * & * * * \\ \mathrm{~ns} & * * * & * * * \\ * & * * * & * * * \\ \mathrm{~ns} & * * * & \mathrm{~ns} \\ \mathrm{~ns} & * * * & * * *\end{array}$

BL: Bottom leaves; ML: Middle leaves; UL: Upper leaves; the small letter is for comparing rootstocks grafting and the capital letter is for comparing leaf orientation; values are the means of three replicate samples. Means followed by different letters within a column and row and under a specific treatment effect are significantly different and separated using the least significant difference (LSD) test; $\mathrm{ns},{ }^{*},{ }^{* * *}$ non-significant or significant at $p<0.05,0.001$, respectively.

Leaves of grafted plants had lower $\mathrm{Na}^{+}$content in younger leaves and higher in older leaves at salinity levels of 6 and $9 \mathrm{dS} \mathrm{m}^{-1}$. The average $\mathrm{Na}^{+}$content of young leaves of plants grafted on rootstock IC-111056 was lower by $19.87 \%$ and $27.50 \%$, respectively than non-grafted plants at $\mathrm{EC}_{\mathrm{iw}} 6$ and $9 \mathrm{dS} \mathrm{m}^{-1}$. On the contrary, the $\mathrm{Na}^{+}$content of older leaves of plants grafted on rootstock IC-111056 was higher by $14.89 \%$ and $13.82 \%$, respectively than non-grafted plants at both salinity levels (Table 5). In grafted plants, the $\mathrm{K}^{+}$content was high in upper and middle leaves and lower in bottom leaves under different salt treatments. Therefore, $\mathrm{K}^{+} / \mathrm{Na}^{+}$ratios of grafted plants were significantly high in upper and middle leaves and low in bottom leaves than non-grafted plants. The plants grafted on rootstock IC-111056 showed significantly higher $\mathrm{K}^{+} / \mathrm{Na}^{+}$ratios in upper and middle leaves compared to non-grafted plants as well as plants grafted on rootstock IC-354557. 


\subsection{Trait Association}

Trait association revealed through Pearson's correlation coefficients indicated that horticultural traits, such as plant height, fruit weight, and fruit yield were significantly positively correlated $(p<0.01)$ with SPAD, $\mathrm{RWC}, \mathrm{K}^{+}$concentration, and $\mathrm{K}^{+} / \mathrm{Na}^{+}$ratios in different plant parts. Conversely, these three traits were significantly negatively associated with antioxidant enzymes (CAT, APX, SOD, and POX), organic osmolyte (proline), and $\mathrm{Na}^{+}$content in root, shoot, and leaves. This suggested that $\mathrm{K}^{+}$partitioning may assist in survival under salinity stress. Furthermore, the total soluble sugar (TSS) showed a negative association with APX and $\mathrm{Na}^{+}$concentration in plant shoots (Figure 1). Proline accumulation showed a strong positive association with antioxidant enzymes (CAT, APX, $\mathrm{SOD}$, and $\mathrm{POX}$ ) and $\mathrm{Na}^{+}$compartmentation in organs and a strong negative association with $\mathrm{K}^{+}$concentration and $\mathrm{K}^{+} / \mathrm{Na}^{+}$ratios in different organs. However, the SPAD index and RWC showed a reverse trend, i.e., they were negatively associated with antioxidant enzymes (CAT, APX, SOD, and POX) and $\mathrm{Na}^{+}$compartmentation and positively associated with $\mathrm{K}^{+}$concentration and $\mathrm{K}^{+} / \mathrm{Na}^{+}$ratios in different organs.

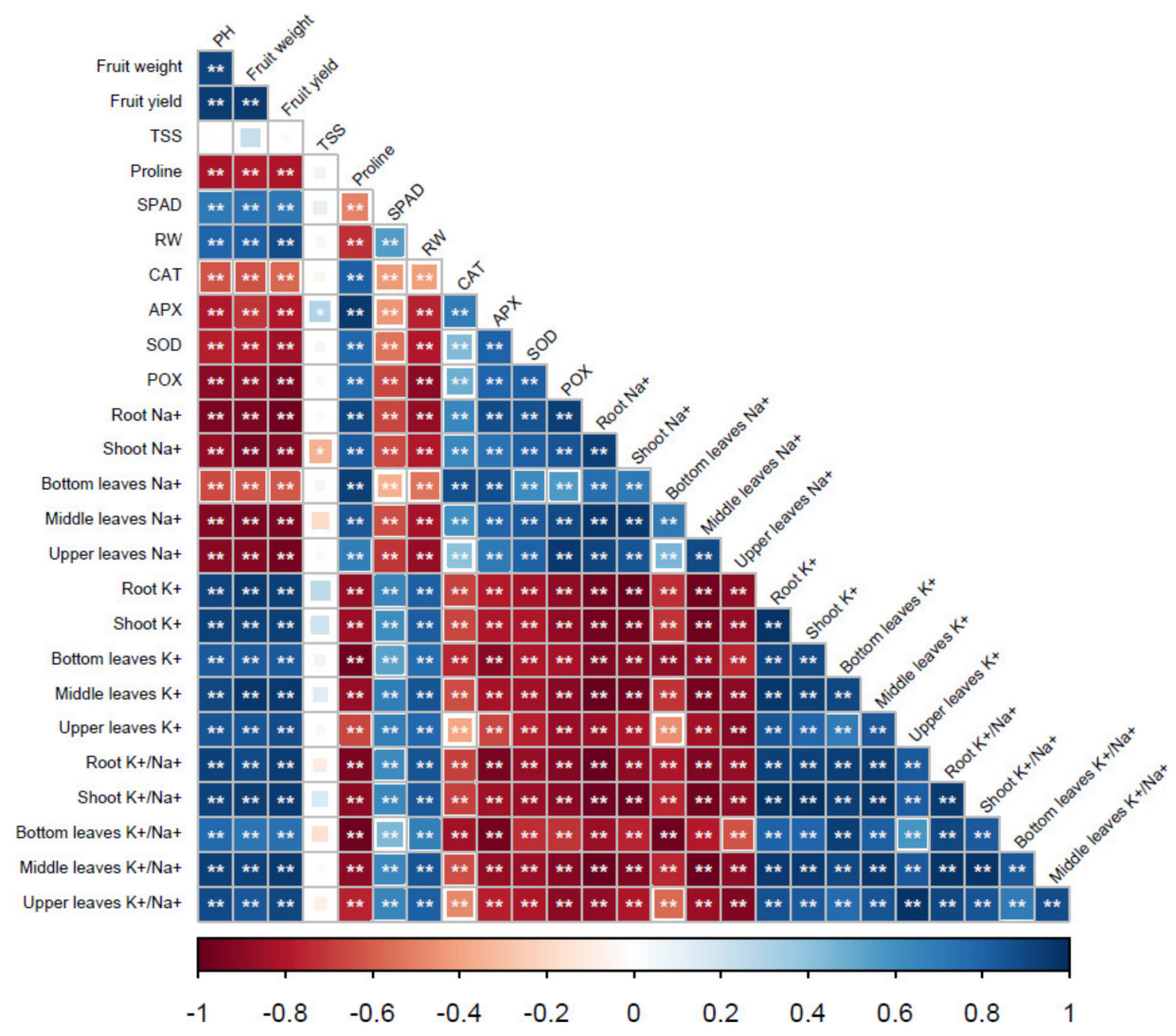

Figure 1. Association between horticultural and biochemical traits of tomato under saline environment.

\section{Discussion}

It is a well-documented fact that plant growth and yield decrease with the increasing salt concentrations. Grafting of salt sensitive plants on tolerant rootstocks provides an alternate and/or complementary mechanism to improve stress tolerance and economic yield. In the present study, tomato plants grafted on two different eggplant rootstocks produced more fruit weight and yield per plant than non-grafted plants under saline water irrigation $\left(\mathrm{EC}_{\mathrm{iw}} 6\right.$ and $\left.9 \mathrm{dS} \mathrm{m} \mathrm{m}^{-1}\right)$. The grafting of salt sensitive tomato plants on salt tolerant eggplant rootstocks improved the salt tolerance of tomato plants through a combination of physiological and biochemical factors. In this study, the average fruit weight and TSS content in tomato under saline environment were determined by scion-rootstock 
interactions. Moreover, these changes correlated with morphological adaptations that allow survival under the higher salt concentrations.

Semiz et al. [34] also reported the enhanced tomato yield under elevated salinity levels in grafted plants. The salt tolerance of grafted plants among various rootstockscion combinations was attributed towards the ionic tolerance at 50 and $75 \mathrm{mM} \mathrm{NaCl}$ in comparison with the lower salinity of $25 \mathrm{mM} \mathrm{NaCl}[22,23]$. The grafted cucumber on bottle gourd rootstock showed less decrease in yield than non-grafted plants with increasing salinity [35]. The plants grafted on rootstock IC-111056 produced more fruit and yield than plants grafted on IC-354557, indicating that the response of grafting combinations on the fruit yield of tomato also depends on the rootstock genotype. Moreover, the effect of both the rootstock genotype and salinity levels on the yield of grafted tomato plants was reported by Savvas et al. [36]. Numerous reports are available that show the enhanced tolerance of grafted Solanaceae crops under saline conditions than self-rooted plants [37-39].

In grafted plants, total soluble solids (TSS) in fruits were higher in plants treated with $6 \mathrm{dS} \mathrm{m}^{-1}$ saline irrigation, but decreased at $9 \mathrm{dS} \mathrm{m}^{-1}$ treatment compared to control. Savvas et al. [36] and Di Gioia et al. [21] observed no effect of grafting combinations on the TSS content, while Rouphael et al. [40] and Turhan et al. [41] reported a reduction in TSS content in grafted tomato plants than non-grafted plants. Several other studies reported decreased soluble solids in plants grafted on different rootstocks [42-45].

In the present study, the relative water content (RWC) and SPAD index were significantly affected by different salinity levels. Although the RWC was generally lower under salinity, the grafted plants displayed higher RWC than non-grafted plants, indicating that the rootstocks contributed to the maintenance of water uptake under salt stress. Herein, we observed $27.08 \%$ reduction in leaf RWC under salinity. Similarly, Tanveer et al. reported that salinity negatively affected the RWC of tomato leaves [46]. However, no significant effect of salinity on leaf RWC was observed in tomato and cucumber grafted on different rootstocks [22,35], which is probably due to the osmotic adjustment [35]. On the contrary, Santa-Cruz et al. [47] observed 35\% increased leaf water content under saline conditions in grafted plants, where scion had a salt-induced character.

Saline toxicity caused a significant decline in chlorophyll content, measured as the SPAD index in non-grafted plants than grafted plants. The SPAD index was higher in plants grafted on the two rootstocks than non-grafted plants, where plants grafted on rootstock IC-111056 displayed more leaf greenness. Colla et al. [48] observed that cucumber plants grafted on Affyne rootstock had high chlorophyll content (SPAD index) than non-grafted plants under salinity stress. A consistent decrease in chlorophyll content and RWC was observed in three cultivars of walnut under saline water irrigation [49]. This reduction in chlorophyll content may be due to the ion accumulation and functional distress of the chlorophyll synthesizing machinery $[50,51]$.

Salinity stress negatively affects various physiological and metabolic processes, leading to the generation of reactive oxygen species (ROS), which could seriously disrupt cellular homeostasis and plant metabolism [52]. To avoid or tolerate these effects, plant cells over synthesize certain osmolytes, especially proline which mainly regulates osmoticum in addition to the stabilization of proteins/membranes [53]. Furthermore, antioxidant enzymes prevent the accumulation of the toxic ROS or detoxify them to minimize the oxidative damage. In this study, proline accumulation increased significantly with the increase in salinity level of both rootstocks of grafted plants compared to non-grafted plants. Generally, the osmolytes, such as proline, sucrose, and glycine betaine increase under salt stress to protect the plants by maintaining cell-homeostasis [54,55]. Grafted plants of cucumber [56,57] and tomato [58] have better salt tolerance due to the high amount of soluble sugar and proline content under salinity.

The antioxidant enzymes help the plants overcome the salt-induced oxidative stress [59]. In the present study, the activities of antioxidant enzymes CAT, APX, SOD, and POX increased with the salinity level in rootstock-grafted plants. These enzymes, CAT, and SOD, in rootstock-grafted plants might detoxify the generated ROS since these two enzymes are 
the first to control the generation of reactive species, and thus protect the cells [60]. Grafted cucumber plants have lower $\mathrm{H}_{2} \mathrm{O}_{2}$ content along with higher activity of CAT, SOD, and POD [61] under salt stress. Similarly, at higher levels of $\mathrm{Ca}\left(\mathrm{NO}_{3}\right)_{2}$, grafted tomato plants had lower $\mathrm{O}^{2-}, \mathrm{H}_{2} \mathrm{O}_{2}$, MDA contents and high POD, CAT, and SOD activities than selfrooted plants [62]. Therefore, the higher expression of antioxidant enzymes in rootstocks IC-111056 and IC-354557 of grafted tomato plants may be responsible for their enhanced salt tolerance.

The ability of plants to inhibit the translocation of ions between the root and shoot is the main factor for the enhanced salt tolerance [63], which in grafted vegetables has often been correlated with lower ionic ratio in the shoots. In tomato, we observed that the $\mathrm{Na}^{+}$content was lower in the grafted plant's root and shoot than non-grafted plants. This indicates that both rootstocks enhanced the plant's capacity to exclude $\mathrm{Na}^{+}$with rootstock IC-111056 found to be superior to rootstock IC-354557. Colla et al. [48] reported less aerial $\mathrm{Na}^{+}$content in grafted plants than non-grafted cucumber, suggesting the higher $\mathrm{Na}^{+}$ exclusion capacity of the grafted plants. The lower $\mathrm{Na}^{+}$content in the upper parts of grafted tomato plants was also reported by Estan et al. [22] and Martinez-Rodriguez et al. [23].

In contrast to $\mathrm{Na}^{+}$, the root and shoot of grafted plants have high $\mathrm{K}^{+}$concentration than non-grafted plants. Interestingly, in both types of plants, shoots had higher $\mathrm{K}^{+} / \mathrm{Na}^{+}$ ratios than roots under both saline treatments. Comparatively, no effect was seen in $\mathrm{K}^{+}$ level of leaves by Savvas et al. [36] and He et al. [64]. The $\mathrm{K}^{+}$homeostasis is also genotype and species dependent in defining salinity stress tolerance [65]. The high $\mathrm{K}^{+} / \mathrm{Na}^{+}$ratios in the grafted tomato plants may indicate an increased level of salinity tolerance through $\mathrm{K}^{+}$ homeostasis in the grafted plants $[57,66]$.

Ion partitioning in different leaf orientations, i.e., bottom (older), medium, and upper (young) leaves was analyzed. Ion accumulation and subsequent partitioning are part of the salt tolerance mechanisms, in which all of the plants greatly employ to ease the toxic effect of salt [59]. In our study, the $\mathrm{Na}^{+}$content was lower in the middle and upper leaves, but high in bottom leaves of the rootstock grafted tomato plants compared with non-grafted plants, indicating the role of rootstock in salt exclusion. Furthermore, the uptake of $\mathrm{K}^{+}$and $\mathrm{K}^{+} / \mathrm{Na}^{+}$ratios was higher in upper leaves followed by the middle and bottom leaves in both non-grafted and grafted plants. However, the grafted plants had better $\mathrm{K}^{+}$uptake with high $\mathrm{K}^{+} / \mathrm{Na}^{+}$ratios than non-grafted plants, particularly in the upper leaves, indicating the potential of grafted plants to limit the ion imbalances under salt stress condition. This revealed $\mathrm{Na}^{+}$partitioning within the shoot tissue of grafted plant by the dint of the lowering $\mathrm{Na}^{+}$movement towards the younger leaves and inclusion of $\mathrm{Na}^{+}$in the bottom leaves for tackling excess $\mathrm{Na}^{+}$toxicity, as has been reported in previous studies [67]. Due to this partitioning, grafted plants were able to maintain favorable $\mathrm{K}^{+} / \mathrm{Na}^{+}$ ratios in the actively growing leaves enhancing their salt tolerance. Earlier studies also reported higher $\mathrm{K}^{+} / \mathrm{Na}^{+}$ratios in the upper leaves or aerial parts of the grafted plants than non-grafted plants [21,36]. In saline environments, the equilibrium of high $\mathrm{K}^{+} / \mathrm{Na}^{+}$ratios in grafted plants are generally due to the enhanced uptake of $\mathrm{K}^{+}$in rootstocks [68]. The maintenance of high $\mathrm{K}^{+} / \mathrm{Na}^{+}$ratios in plant tissue and cytosols is the best strategy to adapt under salt stress, through the regulation of uptake and transfer of $\mathrm{Na}^{+}$[69]. In addition, limiting ion accumulation in young tissues is important for salt tolerance [70,71]. Salt stress alters the $\mathrm{K}^{+}$efflux in both roots and shoots due to the salt stress -induced high $\mathrm{Na}^{+}$ influx through membrane depolarization [72]. Briefly, the capacity of plants to maintain the cytosolic $\mathrm{K}^{+} / \mathrm{Na}^{+}$ratios through $\mathrm{K}^{+}$accumulation or restricting $\mathrm{Na}^{+}$in leaves, helps in balancing the threshold level of $\mathrm{K}^{+}$, and thus better plant performance under salt stress. As $\mathrm{K}^{+}$has an important role in osmoregulation through the accumulation of solutes and osmolytes [73], this in turn lowers the osmotic potential of the cell, and thus the water status of cell is maintained against turgor pressure, finally, enabling the plants to overcome the stress effects.

The available reports demonstrate the correlation of tomato fruit yield to grafting per se [74]. This positive correlation may be due to the improved water use efficiency of the 
rootstocks used for grafting $[75,76]$ or enhanced scion vigor $[77,78]$. The combination of any or all of the mentioned mechanisms could contribute towards increasing the crop yield of grafted tomato plants under salt stress.

\section{Conclusions}

Soil salinity reduces tomato productivity to a large extent. In this study, tomato plants grafted on rootstocks IC-111056 and IC-354557 demonstrated better salt stress tolerance in comparison with non-grafted plants. The grafted plants maintained higher relative water content and antioxidant enzyme activities, along with the accumulation of osmolyte proline to balance the reduced damage caused by oxidative stress and desiccation. Furthermore, the grafted plants had more $\mathrm{K}^{+}$ions and high $\mathrm{K}^{+} / \mathrm{Na}^{+}$ratios in younger leaves than older leaves, demonstrating that the rootstock may confer $\mathrm{Na}^{+}$exclusion and $\mathrm{K}^{+}$retention properties to the tomato scion, thereby enhancing the salt tolerance ability of grafted plants. This may be one of the key mechanisms of salt tolerance in the grafted tomato plants. From these results, it could be summarized that the use of appropriate salt tolerant rootstock for vegetable grafting could provide an alternate approach to increase the yield of high performing, salt-sensitive variety in salt affected soils.

Supplementary Materials: The following are available online at https:/ / www.mdpi.com/article/ 10.3390/agriculture12020183/s1. Tables S1-S3: Analysis of variance (ANOVA) for yield components and physiological parameters of tomato.

Author Contributions: Conceptualization, S.K.S., R.K. and P.C.S.; data curation, A.M., A.K. (Arvind Kumar), H.K., G.K., A.K.R., R.K., A.K. (Ashwani Kumar), A.B. and B.S.; formal analysis, A.K. (Arvind Kumar), H.K., G.K., R.K. and A.K. (Ashwani Kumar); funding acquisition, S.K.S. and P.C.S.; investigation, S.K.S., A.M., A.K. (Arvind Kumar), H.K., G.K., A.K.R., R.K. and A.K. (Ashwani Kumar); methodology, S.K.S., A.M., A.K. (Arvind Kumar), G.K., A.K.R, R.K., P.C.S., A.B. and P.K.; project administration, S.K.S. and P.C.S.; resources, S.K.S., A.B., B.S. and P.K.; supervision, A.K. (Arvind Kumar) and P.C.S.; validation, S.K.S., A.M., A.K. (Arvind Kumar), H.K., G.K., A.K.R., P.C.S., A.K. (Ashwani Kumar), A.B., B.S. and P.K.; writing-original draft- S.K.S., A.M., G.K. and R.K.; writingreview and editing, A.K. (Arvind Kumar), H.K., A.K.R., R.K., P.C.S., A.K. (Ashwani Kumar), A.B., B.S. and P.K.; draft finalization, P.K., A.M., S.K.S., A.B., A.K. and B.S. All authors have read and agreed to the published version of the manuscript.

Funding: This study received no external funding.

Institutional Review Board Statement: Not applicable.

Informed Consent Statement: Not applicable.

Data Availability Statement: The datasets generated for this study are available on request from the principal or corresponding authors.

Acknowledgments: The authors are highly thankful to the ICAR-Central Soil Salinity Research Institute, Karnal for providing all of the facilities.

Conflicts of Interest: The authors declare no conflict of interest.

\section{References}

1. Mandal, S.; Raju, R.; Kumar, A.; Kumar, P.; Sharma, P.C. Current status of research, technology response and policy needs of salt-affected soils in India-A review. Ind. Soc. Coast. Agric. Res. 2018, 36, 40-53.

2. Shrivasata, P.; Kumar, R. Soil salinity: A serious environmental issue and plant growth promoting bacteria as one of the tools for its alleviation. Saudi J. Biol. Sci. 2015, 22, 123-131. [CrossRef] [PubMed]

3. Machado, R.M.; Serralheiro, R.P. Soil salinity: Effect on vegetable crop growth. Management practices to prevent and mitigate soil salinization. Horticulturae 2017, 3, 30. [CrossRef]

4. Maas, E.V.; Grattan, S.R. Crop yields as affected by salinity. Agric. Drain. 1999, 38, 55-110.

5. Foolad, M.R. Recent advances in genetics of salt tolerance in tomato. Plant Cell Tissue Organ. Cult. 2004, 76, 101-119. [CrossRef]

6. Kashyap, S.P.; Prasanna, H.C.; Kumari, N.; Mishra, P.; Singh, B. Understanding salt tolerance mechanism using transcriptome profiling and de novo assembly of wild tomato Solanum chilense. Sci. Rep. 2020, 10, 15835. [CrossRef] 
7. Hajer, A.S.; Malibari, A.A.; Al-Zahrani, H.S.; Almaghrabi, O.A. Responses of three tomato cultivars to sea water salinity 1. Effect of salinity on the seedling growth. Afr. J. Biotechnol. 2006, 5, 855-861.

8. Khavari-Nejad, R.A.; Chaparzadeh, N. The effects of $\mathrm{NaCl}$ and $\mathrm{CaCl}_{2}$ on photosynthesis and growth of alfalfa plants. Photosynthetica 1998, 35, 461-466. [CrossRef]

9. Giuffrida, F.; Martorana, M.; Leonardi, C. How sodium chloride concentration in the nutrient solution influences mineral composition of tomato and fruits. Hortic. Sci. 2009, 44, 707-711. [CrossRef]

10. Singh, H.; Kumar, P.; Kumar, A.; Kyriacou, M.C.; Colla, G.; Rouphael, Y. Grafting tomato as a tool to improve salt tolerance Agronomy 2020, 10, 263. [CrossRef]

11. Zheng, Q.; Liu, J.; Liu, R.; Wu, H.; Jiang, C.; Wang, C.; Guan, Y. Temporal and spatial distributions of sodium and polyamines regulated by brassinosteroids in enhancing tomato salt resistance. Plant Soil 2016, 400, 147-164. [CrossRef]

12. Al-Harbi, A.; Hejazi, A.; Al-Omran, A. Responses of grafted tomato (Solanum lycopersicon L.) to abiotic stresses. Saudi J. Biol. Sci. 2016, 24, 1274-1280. [CrossRef]

13. Sauvage, C.; Segura, V.; Bauchet, G.; Stevens, R.; Do, P.T.; Nikoloski, Z.; Fernie, A.R.; Causse, M. Genome-wide association in tomato reveals 44 candidate loci for fruit metabolic traits. Plant Physiol. 2014, 165, 1120-1132. [CrossRef]

14. Cuartero, J.; Bolarin, M.C.; Asins, M.J.; Moreno, V. Increasing salt tolerance in the tomato. J. Exp. Bot. 2006, 57, 1045-1058 [CrossRef]

15. Zhao, X.; Simonne, E.H. Introducing grafting technology to the Florida tomato industry: Potential benefits and challenges. In Florida Tomato Institute Proceedings; Florida Tomato Institute: Naples, FL, USA, 2008.

16. Rouphael, Y.; Venema, J.H.; Edelstein, M.; Savvas, D.; Colla, G.; Ntatsi, G.; Ben-Hur, M.; Kumar, P.; Schwarz, D. Grafting as a tool for tolerance of abiotic stress. In Vegetable Grafting: Principles and Practices; Colla, G., Schwarz, D., Pérez-Alfocea, F., Eds.; CAB International: Wallingford, UK, 2017; pp. 171-215.

17. Wang, Q.; Men, L.; Gao, L.; Tian, Y. Effect of grafting and gypsum application on cucumber (Cucumis sativus L.) growth under saline water irrigation. Agric. Water Manag. 2017, 188, 79-90. [CrossRef]

18. Kawaguchi, M.; Taji, A.; Backhouse, D.; Oda, M. Anatomy and physiology of graft incompatibility in solanaceous plants. J. Hortic. Sci. Biotechnol. 2008, 83, 581-588. [CrossRef]

19. King, S.R.; Davis, A.R.; Zhang, X.; Crosby, K. Genetics, breeding and selection of rootstocks for Solanaceae and Cucurbitaceae. Sci. Hortic. 2010, 127, 106-111. [CrossRef]

20. Keatinge, J.D.H.; Lin, L.J.; Ebert, A.W.; Chen, W.Y.; Hughes, J.A.; Luther, G.C.; Wang, J.F.; Ravishankar, M. Overcoming biotic and abiotic stresses in the Solanaceae through grafting: Current status and future perspectives. Biol. Agric. Hortic. 2014, 30, $272-287$. [CrossRef]

21. Di Gioia, F.; Signore, A.; Serio, F.; Santamaria, P. Grafting improves tomato salinity tolerance through sodium partitioning within the shoot. Hortic. Sci. 2013, 48, 855-862. [CrossRef]

22. Estan, M.T.; Martinez-Rodriguez, M.M.; Perez-Alfocea, F.; Flowers, T.J.; Bolarin, M.C. Grafting raises the salt tolerance of tomato through limiting the concentration of sodium and chloride to the shoot. J. Exp. Bot. 2005, 56, 703-712. [CrossRef]

23. Martinez-Rodriguez, M.M.; Estan, M.T.; Moyano, E.; Garcia-Abellan, J.O.; Flores, F.B.; Campos, J.F.; Al-Azzawi, M.J.; Flowers, T.J.; Bolarin, M.C. The effectiveness of grafting to improve salt tolerance in tomato when an 'excluder' genotype is used as scion. Environ. Exp. Bot. 2008, 63, 392-401. [CrossRef]

24. Bahadur, A.; Rai, N.; Kumar, R.; Tiwari, S.K.; Singh, A.K.; Rai, A.K.; Singh, U.; Patel, P.K.; Tiwari, V.; Rai, A.B.; et al. Grafting tomato on eggplant as a potential tool to improve waterlogging tolerance in hybrid tomato. Veg. Sci. 2014, 42, 82-87.

25. Weatherley, P.E. Studies in the water relations of the cotton plant. I. The field measurement of water deficits in leaves. New Phytol. 1950, 49, 81-87. [CrossRef]

26. Bates, L.S.; Walden, R.P.; Teare, I.D. Rapid determination of proline for water stress studies. Plant Soil 1973, 39, 205-207. [CrossRef]

27. Chawla, S.; Jain, S.; Jain, V. Salinity induced oxidative stress and antioxidant system in salt-tolerant and salt-sensitive cultivars of rice (Oryza sativa L.). J. Plant Biochem. Biotechnol. 2013, 22, 27-34. [CrossRef]

28. Nishikimi, M.; Rao, N.A.; Yagi, K. The occurrence of superoxide anion in the reaction of reduced phenazinemethosulfate and molecular oxygen. Biochem. Biophys. Res. Commun. 1972, 46, 849-854. [CrossRef]

29. Nakano, Y.; Asada, K. Hydrogen peroxide is scavenged by ascorbate specific peroxidase in spinach chloroplasts. Plant Cell Physiol. 1981, 22, 867-880.

30. Shannon, L.M.; Kay, E.; Lew, J.Y. Peroxidase izoenzymes from horse radish roots. J. Biol. Chem. 1966, 241, 2166-2172. [CrossRef]

31. Aebi, H. Catalase in vitro. Methods Enzymol. 1984, 105, 121-126.

32. R Core Team. R: A Language and Environment for Statistical Computing; R Core Team: Vienna, Austria, 2016; Available online: https:/ / www.R-project.org/ (accessed on 1 July 2021).

33. Wei, T.; Simko, V. R Package "corrplot": Visualization of a Correlation Matrix. 2021. Available online: https://github.com/ taiyun/corrplot (accessed on 15 July 2021).

34. Semiz, G.D.; Suarez, D.L. Tomato salt tolerance: Impact of grafting and water composition on yield and ion relations. Turk. J. Agric. For. 2015, 39, 876-886. [CrossRef]

35. Huang, Y.; Tang, R.; Cao, Q.L.; Bie, Z.L. Improving the fruit yield and quality of cucumber by grafting onto the salt tolerant rootstock under $\mathrm{NaCl}$ stress. Sci. Hortic. 2009, 122, 26-31. [CrossRef] 
36. Savvas, D.; Savva, A.; Ntatsi, G.; Ropokis, A.; Karapanos, I.; Krumbein, A.; Olympios, A. Effects of three commercial rootstocks on mineral nutrition, fruit yield, and quality of salinized tomato. J. Plant Nutr. Soil Sci. 2011, 174, 154-162. [CrossRef]

37. Colla, G.; Rouphael, Y.; Leopardi, C.; Bie, Z. Role of grafting in vegetable crops grown under saline conditions. Sci. Hortic. 2010, 127, 147-155. [CrossRef]

38. Edelstein, M.; Ben-Hur, M.; Cohen, R.; Burger, Y.; Ravina, I. Boron and salinity effects on grafted and non-grafted melon plants. Plant Soil 2005, 269, 273-284. [CrossRef]

39. Goreta, S.; Bucevic-Popovic, V.; Vuletin-Selak, G.; Pavela-Vrancic, M.; Perica, S. Vegetative growth, superoxide dismutase activity, and ion concentration of salt stressed watermelon as influenced by rootstock. J. Agric. Sci. 2008, 146, 695-704. [CrossRef]

40. Rouphael, Y.; Schwarz, D.; Krumbein, A.; Colla, G. Impact of grafting on product quality of fruit vegetables. Sci. Hortic. 2010, 127, 172-179. [CrossRef]

41. Turhan, A.; Ozmen, N.; Serbeci, M.S.; Seniz, V. Effects of grafting on different rootstocks on tomato fruit yield and quality. Hortic. Sci. 2011, 38, 142-149. [CrossRef]

42. Lopez-Galarza, S.; San Bautista, A.; Perez, D.M.; Miguel, A.; Baixauli, C.; Pascual, B.; Maroto, J.V.; Guardiola, J.L. Effects of grafting and cytokinin-induced fruit setting on colour and sugar-content traits in glasshouse-grown triploid watermelon. J. Hortic. Sci. Biotechnol. 2004, 79, 971-976. [CrossRef]

43. Pogonyi, A.; Pek, Z.; Helyes, L.; Lugasi, A. Effect of grafting on the tomato's yield, quality and main fruit components in spring forcing. Acta Aliment. 2005, 34, 453-462. [CrossRef]

44. Qaryouti, M.M.; Qawasmi, W.; Hamdan, H.; Edwan, M. Tomato fruit yield and quality as affected by grafting and growing system. Acta Hortic. 2007, 741, 199-206. [CrossRef]

45. Alexopoulos, A.; Kondylis, A.; Passam, H.C. Fruit yield and quality of watermelon in relation to grafting. J. Food Agric. Environ. 2007, 5, 178-179.

46. Tanveer, K.; Gilani, S.; Hussain, Z.; Ishaq, R.; Adeel, M.; Ilyas, N. Effect of salt stress on tomato plant and the role of calcium. J. Plant Nutr. 2020, 43, 28-35. [CrossRef]

47. Santa-Cruz, A.; Martinez-Rodriguez, M.M.; Perez-Alfocea, F.; Romero-Aranda, R.; Bolarin, M.C. The rootstock effect on the tomato salinity response depends on the shoot genotype. Plant Sci. 2002, 162, 825-831. [CrossRef]

48. Colla, G.; Rouphael, Y.; Jawad, R.; Kumar, P.; Rea, E.; Cardarelli, M. The effectiveness of grafting to improve $\mathrm{NaCl}_{\text {and }} \mathrm{CaCl}_{2}$ tolerance in cucumber. Sci. Hortic. 2012, 164, 380-391. [CrossRef]

49. Akça, Y.; Samsunlu, E. The effect of salt stress on growth, chlorophyll content, proline and nutrient accumulation, and K/Na ratio in walnut. Pak. J. Bot. 2012, 44, 1513-1520.

50. Molazem, D.; Qurbanov, E.M.; Dunyamaliyev, S.A. Role of proline, Na and chlorophyll content in salt tolerance of corn (Zea mays L.). Amer. Eurasian J. Agric. Environ. Sci. 2010, 9, 319-324.

51. Nawaz, K.; Talat, A.I.; Hussain, K.; Majeed, A. Induction of salt tolerance in two cultivars of sorghum (Sorghum bicolor L.) by exogenous application of proline at seedling stage. World Appl. Sci. J. 2010, 10, 93-99.

52. Charles, J.B.; Henning, R.; Nicolas, S.; Dirk, R.; Kiran, R.P.; Jens, N.; Joachim, S.; Liu, J.L.; Alisdair, R.F.; Lee, J.S. The metabolic response of heterotrophic Arabidopsis cells to oxidative stress. Plant Physiol. 2007, 143, 312-325.

53. Rhodes, D.; Nadolska-Orczyk, A.; Rich, P.J. Salinity, osmolytes and compatible solutes. In Salinity: Environment-Plants-Molecules; Lauchli, A., Luttge, U., Eds.; Springer: Dordrecht, The Netherlands, 2002; pp. 181-204.

54. Zhu, J.K. Plant salt stress. In Encyclopedia of Life Sciences; John Wiley and Sons, Ltd.: Hoboken, NJ, USA, 2001. [CrossRef]

55. Huang, Y.; Bie, Z.L.; Liu, Z.X.; Zhen, A.; Wang, W.J. Exogenous proline increases the salt tolerance of cucumber by enhancing water status and peroxidase enzyme activity. Soil Sci. Plant Nutr. 2009, 55, 698-704. [CrossRef]

56. Yang, L.F.; Zhu, Y.L.; Hu, C.M.; Liu, Z.L.; Wei, G.P. Effects of salt stress on biomass formation and ion partition in hydroponically cultured grafted cucumber. Acta Bot. Boreali-Occident. Sin. 2006, 26, 2500-2505.

57. Huang, Y.; Zhu, J.; Zhen, A.; Chen, L.; Bie, Z.L. Organic and inorganic solutes accumulation in the leaves and roots of grafted and ungrafted cucumber plants in response to $\mathrm{NaCl}$ stress. J. Food Agric. Environ. 2009, 7, 703-708.

58. Chen, S.F.; Zhu, Y.L.; Liu, Y.L.; Li, S.J. Effects of NaCl stress on activities of protective enzymes, contents of osmotic adjustment substances and photosynthetic characteristics in grafted tomato seedlings. Acta Hortic. Sin. 2005, 32, 609-613.

59. Kaashyap, M.; Ford, R.; Bohra, A.; Kuvalekar, A.; Mantri, N. Improving salt tolerance of chickpea using modern genomics tools and molecular breeding. Curr. Genom. 2017, 18, 557-567. [CrossRef]

60. Scandalios, J.G. Oxygen stress and superoxide dismutases. Plant Physiol. 1993, 101, 7-12. [CrossRef]

61. Zhen, A.; Bie, Z.L.; Huang, Y.; Liu, Z.X.; Li, Q. Effects of scion and rootstock genotypes on the antioxidant defense systems of grafted cucumber seedlings under $\mathrm{NaCl}$ stress. Soil Sci. Plant Nutr. 2010, 56, 263-271. [CrossRef]

62. Zhang, G.W.; Liu, Z.L.; Zhou, J.G.; Zhu, Y.L. Effects of $\mathrm{Ca}\left(\mathrm{NO}_{3}\right)_{2}$ stress on oxidative damage, antioxidant enzymes activities and polyamine contents in roots of grafted and non-grafted tomato plants. Plant Growth Regul. 2008, 56, 7-19. [CrossRef]

63. Hajibagheri, M.A.; Harvey, D.M.R.; Flowers, T.J. Quantitative ion distribution within root cells of salt-sensitive and salt-tolerant maize varieties. New Phytol. 1987, 105, 367-379. [CrossRef]

64. He, Y.; Zhu, Z.; Yang, J.; Ni, X.; Zhu, B. Grafting increases the salt tolerance of tomato by improvement of photosynthesis and enhancement of antioxidant enzymes activity. Environ. Exp. Bot. 2009, 66, 270-278. [CrossRef]

65. Munns, R.; Tester, M. Mechanisms of salinity tolerance. Annu. Rev. Plant Biol. 2008, 59, 651-681. [CrossRef] 
66. Zhu, J.; Bie, Z.L.; Huang, Y.; Han, X.Y. Effect of grafting on the growth and ion contents of cucumber seedlings under NaCl stress. Soil Sci. Plant Nutr. 2008, 54, 895-902. [CrossRef]

67. Albacete, A.; Martínez-Andújar, C.; Ghanem, M.E.; Acosta, M.; Sánchez-Bravo, J.; Asins, M.J.; Cuartero, J.; Lutts, S.; Dodd, I.C.; Perez-Alfocea, F. Rootstock-mediated changes in xylem ionic and hormonal status are correlated with delayed leaf senescence, and increased leaf area and crop productivity in salinized tomato. Plant Cell Environ. 2009, 32, 928-938. [CrossRef]

68. Leonardi, C.; Giuffrida, F. Variation of plant growth and macronutrient uptake in grafted tomatoes and eggplants on three different rootstocks. Eur. J. Hortic. Sci. 2006, 71, 97-101.

69. Shabala, S.; Pottosin, I. Regulation of potassium transport in plants under hostile conditions: Implications for abiotic and biotic stress tolerance. Physiol. Plant. 2014, 151, 257-279. [CrossRef]

70. Sanwal, S.K.; Kumar, A.; Mann, A.; Kaur, G. Differential response of pea (Pisum sativum) genotypes exposed to salinity in relation to physiological and biochemical attributes. Indian J. Agric. Sci. 2018, 88, 149-156.

71. Mann, A.; Kaur, G.; Kumar, A.; Sanwal, S.K.; Singh, J.; Sharma, P.C. Physiological response of chickpea (Cicer arietinum L.) at early seedling stage under salt stress conditions. Legume Res. 2019, 42, 625-632. [CrossRef]

72. Falhof, J.; Pedersen, J.T.; Fuglsang, A.T.; Palmgren, M. Plasma membrane $\mathrm{H}^{+}$-ATPase regulation in the center of plant physiology. Mol. Plant. 2016, 9, 323-337. [CrossRef] [PubMed]

73. Egilla, J.N.; Davies, F.T.; Boutton, T.W. Drought stress influences leaf water content, photosynthesis, and water-use efficiency of Hibiscus rosa-sinensis at three potassium concentrations. Photosynthetica 2005, 43, 135-140. [CrossRef]

74. Rivero, R.M.; Ruiz, J.M.; Romero, L. Role of grafting in horticultural plants under stress conditions. Food Agric. Environ. 2003, 1, $70-74$.

75. Lee, J.M. Cultivation of grafted vegetables I. Current status, grafting methods and benefits. Hortic. Sci. 1994, 29, 235-239. [CrossRef]

76. Ruiz, J.M.; Belakbir, A.; Lopez-Cantarero, I.; Romero, L. Leaf-macronutrient content and yield in grafted melon plants. A model to evaluate the influence of rootstock genotype. Sci. Hortic. 1997, 71, 227-234. [CrossRef]

77. Leoni, S.; Grudina, R.; Cadinu, M.; Madeddu, B.; Carletti, M.G. The influence of four rootstocks on some melon hybrids and a cultivar in greenhouse. Acta Hortic. 1990, 287, 127-134. [CrossRef]

78. Zijlstra, S.; Groot, S.P.C.; Jansen, J. Genotypic variation of rootstocks for growth and production in cucumber; possibilities for improving the root system by plant breeding. Sci. Hortic. 1994, 56, 185-186. [CrossRef] 